\title{
LESÕES DO MEMBRO SUPERIOR NO ESPORTE
}

\author{
SPORTS INJURIES OF THE UPPER LIMB
}

\section{Rogerio Teixeira da Silva}

\section{RESUMO}

As lesões esportivas do membro superior são muito comuns da prática de atividades físicas e, por isso, devem ser estudadas detalhadamente, levando-se em consideração aspectos específicos da modalidades esportiva praticada. Especial atenção deve ser dada à dinâmica da articulação do ombro e toda cintura escapular, pois somente assim poderemos tratar de forma mais adequada os atletas, atuando também na prevenção das recidivas, que podem ocorrer em alguns casos devido ao fato de o atleta procurar sempre o retorno ao mesmo nível esportivo anterior à lesão. Este artigo vai focar principalmente o manejo das lesões tendíneas do membro superior, da fisiopatologia até os novos métodos de tratamento das lesões de maior prevalência na prática esportiva em nosso país.

Descritores - Lesões esportivas; Ombro; Cotovelo; Tendinopatia

\section{INTRODUÇÃO}

As lesões do membro superior no esporte são muito frequentes e, em várias modalidades esportivas, podem exigir do ortopedista um conhecimento mais detalhado da sua fisiopatologia para que o melhor tratamento seja alcançado. Apesar de alguns esportes que causam lesões frequentes no membro superior não serem muito praticados no Brasil (como o caso específico do beisebol, que conta com vasta coleção de trabalhos publicados na literatura médica), modalidades como o tênis, voleibol e handebol, por exemplo, necessitam de grande demanda biomecânica do ombro. E esta demanda faz com que a articulação tenha que ser submetida a forças suprafisiológicas durante boa parte do movimento esportivo - na hora do saque, por exemplo, um tenista amador pode gerar forças de rotação angular da ordem de 7.000 graus por segundo, na fase de aceleração $0^{(1)}$.

\section{ABSTRACT}

Sports injuries of the upper limbs are very common in physical activities, and need to be studied in detail, taking into consideration specific aspects of the types of sports practiced. Special attention should be paid to the dynamics of the shoulder girdle and scapular belt, as this will enable us to treat athletes more adequately, also helping prevent recurrences that can occur in some cases, due to the fact that the athlete always attempts to return to their pre-injury level of sport. This review focuses primarily on the management of upper limb tendon sports injuries, from the physiopathology through to the more common new methods of treatment in sports practice in our country.

Keywords - Sports injuries; Shoulder; Elbow; Tendinopathy

Neste artigo focaremos principalmente a parte de diagnósticos específicos e fisiopatologia, fatores importantes para o entendimento da estratégia de tratamento. Falaremos também sobre tratamentos específicos mais modernos, quando enfocarmos principalmente as tendinopatias, patologias muito frequentes quando tratamos atletas.

\section{Principais lesões do membro superior no esporte:}

Atletas adolescentes

Lesão da epífise distal radial (gymnast's wrist)

Lesão da epífise proximal umeral (Little Leaguer's shoulder)

Osteocondrite do capítulo umeral (cotovelo da liga menor)

Os acromiale

Instabilidades de ombro (menores)

Instabilidades de ombro (multidirecionais)

Hiperfrouxidão articular generalizada

Discinesia escapular

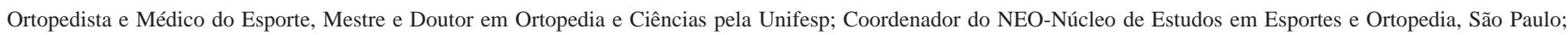
Diretor Médico da Confederação Brasileira de Tênis; Presidente do Comitê de Traumatologia Desportiva da SBOT 2009/2010. 


\begin{abstract}
Atletas adultos
Ombro

Fratura por estresse do úmero distal

Instabilidades do ombro

Síndrome do pinçamento

Lesões do manguito rotador (tendinopatias/rupturas)

Lesão das articulações acromioclaviculares (traumáticas/degeneração articular)

Discinesia escapular
\end{abstract}

\section{Cotovelo}

Epicondilite medial e lateral

Tendinite triceptal

Lesão condral do capítulo umeral

Síndrome compressiva do interósseo posterior

Sinovite do cotovelo

\section{Punho e mão}

Lesão da fibrocartilagem triangular

Tendinite do extensor ulnar do carpo

Sobrecarga óssea do carpo

Fratura por estresse da ulna

\section{LESÕES DO OMBRO NO ESPORTE}

Estima-se que as lesões do ombro representem de $8 \%$ a $13 \%$ do total de lesões esportivas ${ }^{(2)}$. Além do consultório, estas lesões são frequentemente vistas no pronto-atendimento, como demonstrou um estudo feito na Alemanha, com apoio do Ministério da Saúde local, que documentou entre os anos de 1997 a 1999 um total de 7.124 atendimentos a pessoas que sofreram lesões que necessitassem de atendimento médico ${ }^{(3)}$. Do total de lesões relatadas, $3,1 \%$ foram lesões esportivas, e a maioria foi relatada como sendo luxações, torções ou lesões ligamentares (60\%). Vemos, por este trabalho, que as lesões esportivas são importantes de serem estudadas, mesmo quando não somos especialistas em atender atletas somente.

Neste artigo vamos resumir as principais lesões que encontramos no dia a dia, levando-se em conta que uma vasta análise de cada tipo específico de lesão não seria possível.

\section{a) Síndrome do pinçamento subacromial}

É uma patologia frequente do atleta, principalmente aqueles que desempenham mecanismos de arremesso (voleibol, handebol, tênis, entre outros). Temos na prática clínica dois tipos de pinçamento:

a1) Pinçamento subacromial primário: causado pelo impacto do manguito rotador entre a grande tuberosidade e o arco coracoacromial ou a articulação acromio- clavicular. As causas são principalmente anormalidades anatômicas do acrômio (acrômio tipo III), osteófitos subacromiais e artrose acromioclavicular ${ }^{(4,5)}$. Neer classificou o pinçamento em três fases:

Estágio 1: caracterizado por edema e hemorragia na bursa subacromial e no tendão do supraespinal, como resultado de traumas repetitivos (como por exemplo, o movimento de saque do tênis);

Estágio 2: nesta fase, o processo inflamatório produz fibrose e tendinite na porção distal insercional dos tendões;

Estágio 3: é caracterizado pela ruptura parcial ou total do tendão (qualquer um dos componentes do manguito rotador).

Esta classificação, ainda que antiga, dá-nos uma ideia muito boa da evolução e do prognóstico das dores de ombro nos atletas, e deve ser lembrada sempre que se lida com esportistas com dores de ombro. Dependendo da fase que a lesão se encontra, vamos propor um ou outro tratamento, que varia desde o tratamento exclusivamente clínico até a realização de cirurgias corretivas para realização de bursectomia ou acromioplastia, entre outros procedimentos $^{(6)}$.

a2) Pinçamento subacromial secundário: no esporte, este tipo de pinçamento é particularmente importante, principalmente nas populações de esportistas juvenis (na idade de crescimento ósseo). Frequentemente dores na região do ombro, atribuídas ao pinçamento primário, podem ser confundidas com dores secundárias a instabilidades menores, e a atenção deve ser dada para o exame clínico cuidadoso. As principais causas que podem levar a um pinçamento secundário são os quadros menores de instabilidade $\mathrm{e}^{(7)}$, as frouxidões adquiridas após episódios traumáticos e a movimentação inadequada da escápula, conhecida também como discinesia escapular ${ }^{(8)}$.

Do ponto de vista prático, no esportista, o que interessa é pensar nos quadros de pinçamento subacromial e fazer o diagnóstico diferencial com as patologias que podem causar o pinçamento secundário: lesões do manguito rotador, instabilidades glenoumerais e discinesia escapular. Tratar estas patologias em conjunto no esportista que pratica arremesso é imperativo para que tenhamos um bom resultado do tratamento, levando o atleta a um retorno no nível esportivo adequado e desejado.

\section{b) Discinesia escapular}

Apesar de não ser uma patologia isolada, a falta de movimentação adequada da escápula durante os movimentos de arremesso superior no esporte é um dado 
muito importante de ser lembrado quando pensamos em patologias do ombro. Um dos autores que mais estudou esta patologia em esportistas foi Kibler ${ }^{(9)}$, e muitos dos trabalhos falam a respeito da importância do estudo do movimento escapular não somente no tratamento, mas principalmente na prevenção de lesões.

O exame clínico do posicionamento e movimento escapular deve ser feito olhando-se o paciente sem camisa, por trás, para que possamos observar a posição escapular no repouso e também dinamicamente, quando o mesmo realiza movimentos de elevação frontal e lateral dos membros superiores.

As alterações nos movimentos de protração e retração escapular são importantes fatores que geram lesão no ombro durante os movimentos de arremesso no esporte. A perda do movimento completo de retração escapular no tórax faz com que se perca a potência de estabilidade durante a fase de preparação do arremesso, diminuindo o poder de explosão durante a fase de aceleração do golpe. A falha para realização do movimento completo de protração da escápula na parede torácica aumenta a força de desaceleração do ombro no movimento de arremesso, fazendo com que aumente a chance de pinçamentos do manguito rotador - este movimento é especialmente importante porque usualmente estes atletas apresentam grande tensão da cápsula posterior do ombro ${ }^{(10,11)}$.

O tratamento para esta patologia é eminentemente clínico, por meio de adequado programa de reabilitação com os seguintes objetivos: alongamento adequado da cápsula posterior do ombro, equilíbrio de força muscular de rotadores internos e externos do ombro e trabalho específico de fortalecimento e propriocepção da articulação escapulotorácica.

\section{c) Lesões do manguito rotador}

São lesões muito frequentes no esportista de arremesso $^{(12,13)}$. Em nosso país, os principais esportes que causam esta lesão são o tênis e o voleibol.

As tendinopatias (que variam de inflamações a degenerações teciduais) costumam ser mais frequentes na população jovem, enquanto as rupturas (parciais ou totais) são mais vistas na população esportiva acima de 40 anos de idade. Lehman, por exemplo, relatou em um artigo que estudou tenistas nos quais as dores no ombro foram encontradas em populações jovens (12 a 19 anos de idade) com incidência de $24 \%$, enquanto na população acima de 50 anos este número foi ao redor de $50 \%{ }^{(14)}$. Isso se deve ao fato do desgaste e da desidratação a que sofrem as estruturas tendíneas com o avançar da idade, o que explica a importância de se pensar na prevenção nesta população específica.

As lesões de manguito rotador podem ser complicadas de tratar em esportistas, principalmente quando atendemos tenistas profissionais ou amadores que jogam regularmente. Em muitas vezes, o quadro doloroso não corresponde ao que os exames de imagem dizem sobre a lesão anatômica e, vez por outra, deparamo-nos com tenistas com rupturas completas de um tendão (supraespinal, por exemplo) que conseguem jogar normalmente, com praticamente nenhuma dor relatada - este dado é especialmente importante de ser lembrado na população acima de 50 anos de idade, na qual podemos ter um bom resultado no tratamento fisioterápico mesmo quando estamos tratando uma ruptura completa de um único tendão do manguito rotador.

A cirurgia de reparo do tendão do manguito tem demonstrado bons resultados na literatura médica, e pode ser realizada tanto por via artroscópica quanto por via $\operatorname{aberta}^{(15,16)}$. Nossa preferência é pela realização do reparo artroscópico, mas acreditamos que a escolha da técnica deve ser individualizada, e o cirurgião deve ficar confortável com a técnica escolhida. Uma boa cirurgia realizada por via aberta é sem dúvida preferível a uma artroscópica mal realizada.

\section{d) Instabilidade glenoumeral}

As instabilidades também são lesões que podem ser encontradas em esportistas de arremesso, principalmente aquelas que são consideradas adaptações do movimento de arremesso a longo prazo (como as microinstabilidades anteriores, causadas basicamente por déficit de rotação interna do ombro devido a maior tensão da cápsula posterior, o que é muito comum de observarmos no esportista juvenil de tênis, por exemplo). O diagnóstico aqui mais importante de ser feito é se o atleta apresenta ou não déficit de rotação interna glenoumeral (GIRD, do termo em inglês). Esta alteração é caracterizada pela diminuição da rotação interna do ombro, quando comparada com o braço contralateral, e é causada tanto por problemas de partes moles quanto por problemas ósseos ${ }^{(17,18)}$. O importante é que façamos um diagnóstico precoce desta alteração em nossos atletas, pois como as lesões de ombro são frequentes na vida competitiva, a literatura preconiza um trabalho de prevenção sempre que nos depararmos com atletas que apresentem este déficit ${ }^{(19)}$.

Não vamos entrar em detalhes, neste artigo, sobre diferentes tipos de tratamento destas lesões, mesmo porque a discussão não caberia no escopo deste artigo de 
revisão. Vários outros artigos de nossa revista focam a abordagem cirúrgica desta patologia, e o tratamento não é muito diferente do que o manejo das lesões na população não esportista.

\section{e) Lesões do tendão do bíceps proximal e lesões labrais superiores (SLAP)}

Nos últimos anos estamos observando um aumento destas lesões, apesar de recentemente elas serem foco de discussão da literatura internacional. Muito tem-se discutido sobre as lesões proximais do bíceps, incluindo sua classificação. Snyder et al $^{(20)}$ classificaram, inicialmente, a lesão em quatro grupos - hoje em dia, falam-se até em 10 tipos de lesão SLAP, o que particularmente achamos exagerado, levando-se em conta que deve existir uma classificação para guiar o tratamento ${ }^{(21)}$. Os autores que consideram esta classificação mais extensa, descrita por Powell et $a l^{(21)}$, consideram alguns tipos como variações dos tipos originais descritos por Snyder et $\mathrm{al}^{(20)}$.

O diagnóstico clínico nem sempre é fácil, pois muitas patologias podem estar associadas à lesão SLAP no esportista de arremesso. Por isso é que devemos nos valer da ressonância magnética sempre que suspeitamos desta lesão, e com as máquinas mais modernas (2.0, 2.5 e 3.0 Tesla) não necessitamos mais do pedido da ressonância magnética com uso de contraste intra-articular (artrorressonância).

Nos esportistas de arremesso é muito comum encontrar a degeneração da inserção do bíceps proximal, e o cuidado deve existir para que não se tenha sempre uma visão cirúrgica de reparar estas lesões. Quando, porém, a lesão é muito sintomática e o labrum se encontra estável, o reparo cirúrgico deve ser realizado, de preferência, por via artroscópica ${ }^{(22-24)}$.

Outro dado da literatura que merece atenção é a associação da lesão de manguito rotador e da lesão tipo SLAP. Sempre que esta última for sintomática e estiver associada com a ruptura do manguito, o resultado cirúrgico é melhor quando são realizadas as duas cirurgias, para fixação das lesões do labrum e do manguito( ${ }^{(25,26)}$. Apesar de a literatura mostrar estes bons resultados, quando nos deparamos com este mesmo procedimento no atleta profissional, os dados podem não ser tão animadores, e o retorno ao mesmo nível esportivo pode não ser alcançado. Isso se deve ao fato de a extensa demanda de rotação e equilíbrio muscular que o atleta profissional deve ter no seu dia a dia, e não são raros os casos em que a cirurgia abrevia a carreira destes atletas.

\section{f) Lesões da articulação acromioclavicular}

O desgaste da articulação acromioclavicular no esportista de arremesso é bem comum, e muitas vezes nos deparamos com um quadro clínico pouco doloroso associado a grande degeneração radiológica desta articulação (Figura 1). Devemos sempre lembrar que estas alterações radiológicas devem, obrigatoriamente, ser correlacionadas com a clínica do atleta, pois esta última deve prevalecer sobre a primeira.

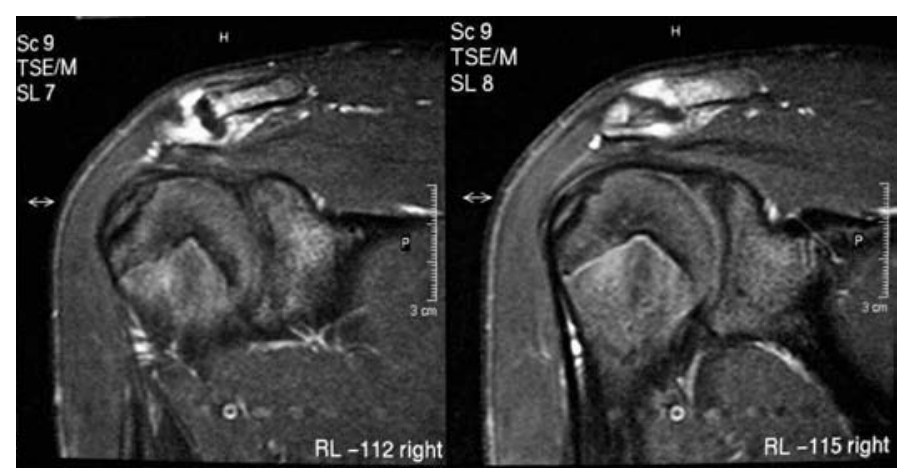

Figura 1 - Note a inflamação da articulação acromioclavicular neste tenista amador de 16 anos de idade. $\mathrm{O}$ atleta não referia nenhuma dor na região, apesar da imagem alterada. O exame foi solicitado para avaliar o manguito rotador

Um dado muito importante que deve ser lembrado é com relação a morfologia do acrômio. Há alguns anos, na cirurgia do ombro, recomendávamos muito mais procedimentos cirúrgicos para deixar o acrômio plano e, hoje, sabemos que nem sempre este procedimento é necessário (Figura 2).

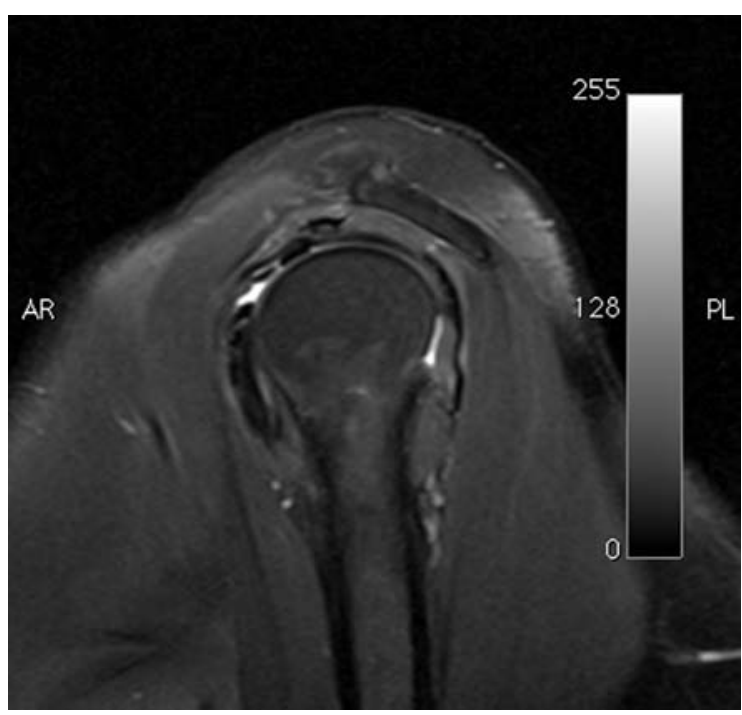

Figura 2 - Note que a forma do acrômio tipo II (curvo) não necessariamente causa impacto nos tendões do manguito rotador (a forma curva se adapta à forma curva desta região da cabeça umeral e tendões que nela estão inseridos) 
Outro dado importante é a distância acrômio-humeral e sua relação com as alterações de posicionamento da escápula. Recentemente, publicamos um trabalho estudando esta relação em tenistas ${ }^{(27)}$. Por meio de testes clínicos e de exames de imagem, observamos que sempre que o espaço subacromial diminui mais do que 20\% quando medido em 0 e em 60 graus de abdução, na ultrassonografia, o tenista tem chance muito grande de apresentar discinesia escapular (Figura 3). Apesar de não termos realizado este estudo longitudinalmente, para observar a relação entre este achado e as lesões de manguito, acreditamos que esta relação deva ser lembrada, para pensarmos na prevenção das lesões de ombro em tenistas juvenis competitivos.

\section{LESÕES DO COTOVELO NO ESPORTE}

As mais frequentes no esporte são as tendinopatias. Não abordaremos lesões traumáticas que serão contempladas em outros artigos.

\section{a) Epicondilite lateral}

Podemos descrever a epicondilite lateral como uma patologia específica da origem da musculatura no epicôndilo lateral, geralmente causada por sobrecarga, em que microlesões são geradas na região de inserção dos tendões extensores (mais frequentemente o extensor radial curto do carpo) do punho, dos dedos e, em menor grau, o extensor radial longo do carpo, além da porção anterior do extensor comum dos dedos.

A dor, geralmente, inicia-se de forma leve e piora progressivamente na região do epicôndilo lateral, po- dendo irradiar para o antebraço, punho, mão e ombro. Conforme se agrava a tendinopatia, tarefas simples como pegar um recipiente com leite ou girar uma maçaneta podem tornar-se difíceis de realizar.

Com relação à prática esportiva, tema principal desta revisão, devemos lembrar sempre da biomecânica do tênis, esporte que mais frequentemente causa esta lesão. Estima-se hoje, segundo relatos da literatura nacional e internacional, que cerca de metade dos tenistas vão ter em algum dia da sua vida esportiva esta lesão, conhecida como tennis elbow ${ }^{(28,29)}$. A empunhadura e os golpes de rebatida do tênis requerem a utilização abrangente dos extensores do antebraço. Os extensores se tornam lesionados à medida que os músculos vão sendo fadigados pela atividade repetitiva.

A técnica incorreta na realização dos golpes é o fator que mais frequentemente causa a epicondilite lateral $^{(30,31)}$. Pacientes que desenvolvem o cotovelo de tenista, de um modo geral, batem seus golpes de $b a$ ckhand com o "cotovelo a frente" sem completar o movimento até o final da batida. Sabe-se que isso provoca estresse excessivo no epicôndilo lateral e atividade anormal da musculatura do antebraço. Outra dica importante da prevenção: se o tenista conseguir jogar com as duas mãos o golpe de backhand isso também previne as lesões. Isso se explica por dois motivos: em primeiro lugar, o atleta dissipa as forças de impacto do golpe nos dois membros superiores; secundariamente, ele também é forçado a rodar mais o tronco na fase de preparação do golpe, o que vai fazer com que a sua energia angular seja maior, evitando o esforço do membro superior dominante isoladamente. Em nossa casuística pessoal,
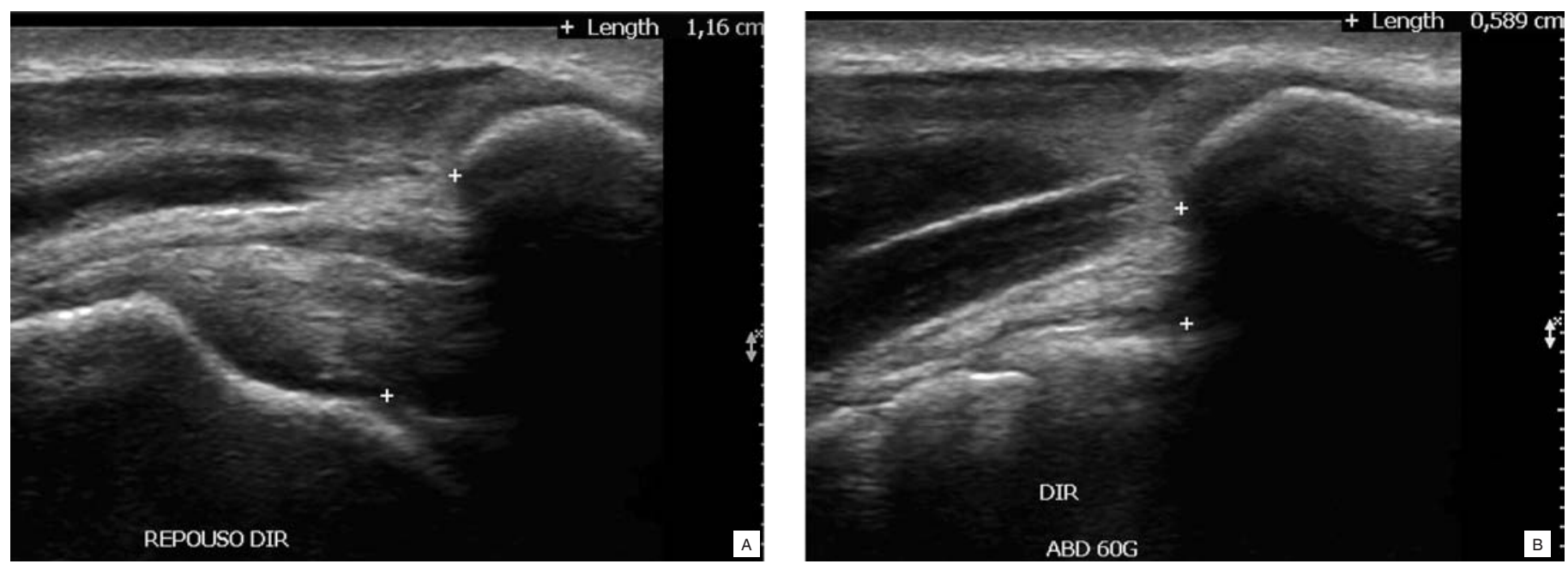

Figura 3 - Exame de ultrassonografia para medida da distância acrômio-humeral. Note que quando o ombro está em repouso (A) existe um bom espaço, porém quando realizamos a abdução a 60 graus (B) este espaço diminui muito em tenistas com discinesia escapular 
do consultório, de um total de 326 tenistas que tiveram epicondilite lateral - tratados de 2004 a 2009 - somente quatro jogavam o backhand com duas mãos.

A epicondilite lateral pode ocorrer pelo uso de equipamentos inadequados. O tamanho da empunhadura da raquete deve ser observada cuidadosamente e personalizada de cada um com o tamanho da mão do atleta, a tensão das cordas da raquete também deve ser medida. Recomendamos, de rotina, que, sempre que o tenista não tenha objeção, em detrimento do rendimento, que sejam utilizadas raquetes de perfil largo. Estas têm a cabeça maior, e área de vibração mínima (sweet spot) maior, o que previne o excesso de vibração na hora da rebatida.

Outra dica importante para o tenista: tensões do encordoamento acima de 57 libras também aumentam a chance de lesão e é por isso que hoje recomendamos que se escolha um equipamento adequado ao seu tipo de jogo e à potência dos golpes que normalmente ele utiliza.

\section{Recomendações gerais para prevenção da epicondilite lateral em tenistas:}

a. Evitar bater as bolas atrasadas

b. Aprender a realizar o complemento dos golpes (saque, direita, esquerda e voleio) de forma adequada

c. Procurar fazer o contato da bola com a cabeça da raquete com o cotovelo posicionado sempre em extensão

d. Preparar bem a rotação de tronco antes do golpe de backhand para poder fazer a rebatida com o cotovelo em extensão

e. Evitar jogar com bolas muito velhas

f. Usar tensões máximas do encordoamento de 56/57 libras

g. Preferir cordas de tripa sintética ou tripa natural

h. Usar raquetes com perfil largo na cabeça, pois elas têm área maior de vibração central mínima (sweet spot)

i. Aproveitar a força de flexão dos joelhos na hora do saque

j. Tentar jogar o backhand com as duas mãos.

\section{Etiopatogenia}

A sobrecarga constante que ocorre em várias atividades esportivas (como o tênis) gera microtraumas nos tendões e nos músculos no epicôndilo lateral, e o fator sobrecarga é o principal mecanismo que gera a lesão. Os microtraumas promovem o surgimento de hiperplasia angiofibroblástica no tendão acometido, como resultado destes estímulos repetitivos crônicos. Esses achados sugerem que a epicondilite crônica constitui uma entidade mais degenerativa que inflamatória. Depois do trauma inicial, estas áreas se lesam com mais frequência, conduzindo a hemorragia e a formação com depósitos de tecido granulosos e de cálcio dentro dos tecidos circunvizinhos ${ }^{(32)}$.

No tênis, exemplo clássico de esporte em que é bem frequente este tipo de patologia, a dor geralmente é exacerbada em decorrência do golpe de backhand e, geralmente, acomete pacientes dos 35 aos 50 anos de idade.

\section{Quadro clínico}

O sintoma principal é dor, que é relatada na parte externa do cotovelo e, ocasionalmente, pode irradiar-se do antebraço para o punho e ombro. A dificuldade para realizar a extensão do antebraço pode ser um sintoma frequente (por causa dos músculos e dos tendões inflamados). Esta dor pode permanecer por semanas ou meses, e não é raro o paciente somente procurar atendimento quando não consegue nem mesmo realizar um aperto de mão. Consideramos a lesão crônica quando os sintomas duram pelo menos três meses.

\section{Diagnóstico clínico}

O diagnóstico clínico leva em consideração vários aspectos relacionados à prática esportiva do tênis. Anamnese bem detalhada sobre o desenvolvimento da disfunção, assim como o tempo de prática do esporte, as formas de aumento e diminuição da dor, movimentos básicos realizados pelo atleta e os testes especiais específicos - teste de Maudsley, teste Cozen e o teste de $\mathrm{Mill}^{(33)}$, praticamente fecham o diagnóstico.

\section{Radiologia}

O exame radiológico simples é realizado de rotina, nas incidências anteroposteriores e perfil. O ultrassom pode ser utilizado, e é um excelente método de imagem para lesões agudas. Em casos crônicos, em que vários tratamentos já foram realizados, a ressonância magnética deve ser solicitada, pois pode trazer dados mais precisos com relação às patologias que podem causar dores semelhantes à epicondilite lateral (como a síndrome de compressão do interósseo posterior) ${ }^{(34)}$. 


\section{Diagnóstico diferencial}

O teste do dedo médio auxilia o diagnóstico diferencial da síndrome do nervo interósseo posterior com a epicondilite lateral. Deve ser realizado com o cotovelo a 90 graus de flexão e punho em posição neutra. Pressiona-se o terceiro dedo, evitando que este faça extensão ativa. O aumento da dor nessa manobra sugere síndrome do interósseo posterior. Outro teste utilizado no diagnóstico diferencial entre essas duas patologias é realizado com a infiltração de 1,0ml de lidocaína no nível do epicôndilo lateral. Nos casos de epicondilite lateral os sintomas desaparecerão, enquanto que, na síndrome do nervo interósseo posterior, os mesmos persistirão ${ }^{(35)}$.

\section{Tratamento clínico e cirúrgico}

Muitos tratamentos foram descritos na literatura para melhorar a dor dos pacientes que sofrem de epicondilite lateral, porém, praticamente todos falam a respeito da população não esportista ${ }^{(36)}$, e isso pode trazer dados conflitantes quando nos deparamos com atletas (basicamente tenistas, população esportiva na qual a lesão é mais prevalente).

Quando nos deparamos com uma lesão aguda, o tratamento é realizado através de analgésicos e antiinflamatórios não hormonais. Essa conduta é sempre indicada junto ao tratamento fisioterápico, pois visa o adequado equilíbrio muscular, analgesia e propriocepção do membro superior como um todo, respeitando o trabalho de adequação ao gesto esportivo praticado ${ }^{(37)}$.

As infiltrações com corticoides podem ser uma saída para a melhora do quadro local de dor, porém a literatura médica deixa bem claro que a maior parte dos efeitos são fugazes e que, a longo prazo, os pacientes que praticam esportes podem voltar a sentir novamente a dor, já que o corticoide não cura a degeneração tecidual que é vista com frequência nos quadros crônicos ${ }^{(38,39)}$.

Outra alternativa de tratamento conservador que podemos usar é a aplicação de ondas de choque. Recentemente, muitos trabalhos com boa evidência científica, falam a favor do uso desta terapia nos casos que não evoluem bem com a fisioterapia convencional ${ }^{(40-42)}$.

A cirurgia da epicondilite lateral só tem espaço para indicação nas lesões crônicas. Quando os sintomas ainda são aparentes por mais de seis meses e houve insucesso do tratamento médico não-cirúrgico e fisioterápico, a cirurgia é indicada. Essa cirurgia consiste na retirada do tecido fibrótico degenerado, localizado na origem dos extensores. A liberação parcial da origem do extensor no epicôndilo lateral do úmero pode ser realizada, mas em esportistas evitamos fazer a liberação e o abaixamento do tendão. Em alguns casos associa-se a ostectomia do epicôndilo, ou até mesmo perfurações ósseas.

Mais recentemente, temos utilizado o tratamento com infiltração de plasma rico em plaquetas (PRP) como alternativa à cirurgia. Estamos, no momento, com 40 casos tratados nos últimos três anos e, em breve, publicaremos os resultados que, em princípio, são promissores, desde que o atleta siga as recomendações de ficar afastado do esporte por pelo menos seis a oito semanas após a infiltração. A terapia com aplicação de PRP procura melhorar o aporte de células plaquetárias no tecido degenerado, visando o reparo e a regeneração das células tendíneas $^{(43)}$. Estas plaquetas, após agregadas, liberam fatores de crescimento plaquetário, dentre eles os fatores vasculares (VEGF), fatores de crescimento ligados ao fibroblasto (FGF), ligados à insulina (IGF), entre outros (Tabela 1).

A terapia com PRP para epicondilite lateral foi descrita pela primeira vez na literatura por Mishra e Pavelko(44), e temos utilizado de rotina para os casos que são refratários ao tratamento conservador e como alternativa à cirurgia ${ }^{(45)}$. Preferimos utilizar a infiltração guiada por ultrassonografia para que a aplicação do PRP seja realizada somente na área lesada, evitando-se assim algumas complicações, como a sinovite reacional, que pode ocorrer se a injeção levar mais volume ao local do que o necessário ${ }^{(46)}$. Isto é muito importante, porque mesmo com adequada centrifugação, após a preparação, o PRP apresenta na sua composição uma pequena quantidade de hemácias (que pode variar de 2 a 4\%). Estas células, em contato com a cobertura sinovial do cotovelo (que está muito próxima da região do tendão extensor comum), podem causar sinovite reacional, que pode prejudicar a reabilitação do paciente. Em nossa casuística, nos casos iniciais, tivemos alguns pacientes que apresentaram sinovites reacionais, que melhoraram após três semanas, mas que nos primeiros dias provocaram um quadro doloroso para a realização de extensão completa do cotovelo. Com a utilização do procedimento guiado pela ultrassonografia, diminuímos a chance deste problema ocorrer, já que podemos observar muito bem a colocação da agulha exatamente no local da lesão.

Outro benefício da ultrassonografia é que podemos utilizar o Doppler para observar a vascularização local, fator este muito importante também na avaliação do processo de regeneração tecidual que ocorre até a oitava semana (Figura 4). Costumamos acompanhar o processo 

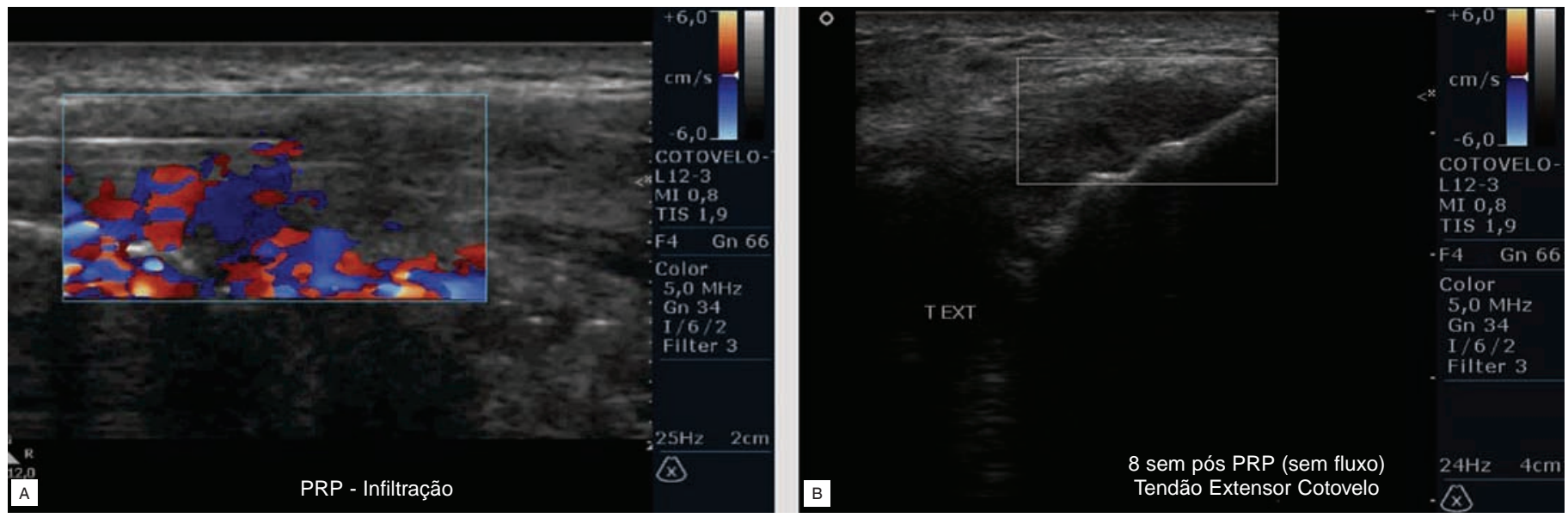

Figura 4 - O uso do doppler na avaliação ultrassonográfica de tendões é importante. Nestes casos ele ajuda no controle da infiltração com $\operatorname{PRP}(\mathrm{A})$ e, para critério de cura, quando mostra ausência de vasos no paciente assintomático (B)

de cicatrização com realização de ultrassonografia com Doppler na quarta, oitava e, quando necessário, na $12^{\mathrm{a}}$ semana. A vascularização normalmente é aumentada no tendão até a quarta semana, diminuindo nos exames de oito semanas, e deve estar ausente no exame com Doppler da $12^{\mathrm{a}}$ semana, quando liberamos o paciente para a prática esportiva competitiva de tênis (Tabela 1).

\section{Tratamento fisioterápico}

A reabilitação da epicondilite lateral ainda é muito controversa, talvez pelo fato de se usar muitas técnicas ao mesmo tempo a fim de recuperar o paciente o mais breve possível. As técnicas mais utilizadas para o tratamento são o uso de ultrassom, ondas curtas, eletroestimulação e gelo ${ }^{(47)}$.

Quando o paciente realiza o tratamento com infiltração de plasma rico em plaquetas, a fisioterapia tem papel fundamental na recuperação tecidual. Utilizamos de roti- na o laser e o ultrassom nas fases iniciais, e já iniciamos precocemente (a partir do segundo dia após a aplicação de PRP) os exercícios leves de extensão e flexão do punho e dedos. A partir daí progride-se para a realização de exercícios excêntricos e propriocepção (a partir da primeira semana) leves, sempre visando a melhora de toda a parte proprioceptiva do membro superior.

A partir da quarta semana, o atleta já se encontra bem para a realização de exercícios específicos do esporte, e podemos inclusive realizar os exercícios com uma raquete mais leve e sem cordas, com ou sem resistência elástica, para incorporar o trabalho de ganho de força muscular.

Os movimentos de direita e esquerda, na clínica de reabilitação, já podem ser realizados com bola mais leve a partir da sexta semana e, a partir daí, iniciamos um programa de reeducação do gesto esportivo. O atleta é liberado definitivamente para jogos e competições entre a $10^{\mathrm{a}}$ e $12^{\mathrm{a}}$ semana.

Tabela 1 - Resumo dos principais fatores de crescimento derivados das plaquetas e suas funções ${ }^{(7)}$

\begin{tabular}{l|l|l}
\hline \multicolumn{1}{c|}{ Fator de crescimento } & \multicolumn{1}{|c}{ Local de onde provém } & \multicolumn{1}{c}{ Função } \\
\hline $\begin{array}{l}\text { TGF beta (transforming growth } \\
\text { factor beta) }\end{array}$ & $\begin{array}{l}\text { Plaquetas, matriz extracelular óssea, ma- } \\
\text { triz cartilaginosa, macrófagos/monócitos e } \\
\text { neutrófilos }\end{array}$ & $\begin{array}{l}\text { Estimula a proliferação da célula mesenquimal não diferenciada; regula a mitogênese } \\
\text { endotelial, do fibroblasto e do osteoblasto; inibe a proliferação dos linfócitos e macrófagos; } \\
\text { regula o efeito mitógeno de outros fatores de crescimento; regula a síntese de colágeno e } \\
\text { a secreção de colagenase }\end{array}$ \\
\hline $\begin{array}{l}\text { bFGF (basic fibroblast growth } \\
\text { factor) }\end{array}$ & $\begin{array}{l}\text { Plaquetas, macrófagos, células mesenqui- } \\
\text { mais, condrócitos e osteoblastos }\end{array}$ & $\begin{array}{l}\text { Promove crescimento e diferenciação de condrócitos e osteoblastos; mitogênico para células } \\
\text { mesenquimais, condrócitos e osteoblastos }\end{array}$ \\
\hline $\begin{array}{l}\text { PDGFa-b (platelet derived growth } \\
\text { factor) }\end{array}$ & $\begin{array}{l}\text { Plaquetas, macrófagos, monócitos, células } \\
\text { mesenquimais, condrócitos, osteoblastos e e } \\
\text { células musculares }\end{array}$ & $\begin{array}{l}\text { Mitogênico para células mesenquimais e osteoblastos; estimula a quimiotaxia e mitogênese } \\
\text { nos fibroblastos, células da glia e células musculares; regula a cãoreção de colagenase e } \\
\text { síntese de colágeno; estimula a quimiotaxia de macrófagos e neutrófilos }\end{array}$ \\
\hline $\begin{array}{l}\text { EGF (epidermal growth factor) } \\
\text { Plaquetas, macrófagos e monócitos }\end{array}$ & $\begin{array}{l}\text { Estimula a quimiotaxia e angiogênese endotelial; regula a secreção de colagenase; estimula } \\
\text { a mitogênese epitelial e mesenquimal }\end{array}$ \\
\hline $\begin{array}{l}\text { VEGF (vascular endothelial } \\
\text { growth factor) }\end{array}$ & Plaquetas e células endoteliais & $\begin{array}{l}\text { Aumenta angiogênese e permeabilidade vascular; estimula a mitogênese das células } \\
\text { endoteliais }\end{array}$ \\
\hline
\end{tabular}




\section{b) Outras lesões tendíneas do cotovelo no esporte}

A epicondilite medial e a tendinopatia do tríceps braquial também são lesões que podem atrapalhar a vida do esportista. Não abordaremos aqui em detalhes estas patologias, somente lembrando que as mesmas podem necessitar de tratamento de longo prazo, principalmente em esportes em que o arremesso com potência é necessário (saque no tenista, arremesso do pitcher no beisebol, ataque no voleibol, entre outros).

O tratamento deve seguir os mesmos caminhos já descritos para a epicondilite lateral, iniciando-se pelas medidas fisioterápicas analgésicas, passando pelo trabalho de fortalecimento muscular e propriocepção. Em casos nos quais o tratamento conservador não obteve bom resultado em um período de três a seis meses, mesmo sendo realizado de forma correta, outras terapias podem ser necessárias, incluindo a terapia por onda de choque, aplicação de plasma rico em plaquetas e cirurgia nos casos refratários a estes tratamentos.

A ruptura do bíceps distal é outra lesão tendínea que podemos encontrar em esportistas, principalmente em halterofilistas, devido à grande força que estes atletas têm que desempenhar para atingir bons resultados. Um dado importante: sempre que nos depararmos com esta lesão, devemos pesquisar se o atleta faz uso ou não de anabolizantes, pois existe relação direta entre o uso destas substâncias e as rupturas deste tendão no cotovelo.

\section{c) Diagnóstico diferencial das tendinopatias do cotovelo no esporte}

\section{Lesões incomuns do ombro e cotovelo no esporte}

Outras lesões do ombro e cotovelo, mais raras, podem acometer os esportistas de arremesso e, dentre elas, destacamos:

a) Lesões nervosas - A mais comum no ombro do esportista é a compressão do nervo supraescapular, mas os nervos axilar e torácico longo também podem sofrer alterações, principalmente crônicas. No cotovelo, a compressão nervosa mais conhecida é a síndrome compressiva do nervo interósseo posterior, que deve ser um diagnóstico diferencial obrigatório dos quadros crônicos.

b) Lesões ósseas por estresse - A sobrecarga óssea mais comum do membro superior é na região do úmero distas e, recentemente, publicamos uma série de casos relatando este diagnóstico nos tenistas ${ }^{(48)}$. Este diagnóstico diferencial é importante de ser lembrado quando o esportista tem dores subagudas e crônicas na região distal do úmero dominante - o tratamento deve ser feito com afastamento temporário do esporte e fisioterapia. Apesar de serem raras, as sobrecargas ósseas também podem ser fonte de dor na região da mão e punho ${ }^{(49)}$.

c) Tumores - Sempre devemos lembrar deste diagnóstico nas dores próximas de articulações, principalmente se a dor é insidiosa e em atletas na fase de crescimento. Os tumores mais comuns são os benignos, mas os malignos também podem acometer principalmente a região proximal do úmero nos atletas adolescentes.

d) Rupturas musculotendíneas - Na região do ombro, uma lesão que não é frequente mas que podemos encontrar no esporte, é a ruptura do músculo peitoral maior, que ocorre em atletas praticantes de levantamento de peso competitivo. Estas lesões também podem estar associadas ao uso de anabolizantes e, em nosso meio, alguns trabalhos já foram descritos sobre a necessidade da cirurgia para correção destes casos em esportistas. No cotovelo, como citamos anteriormente, as rupturas de bíceps distal também podem ser um problema para estes atletas e, geralmente, necessita de procedimentos cirúrgicos para sua correção adequada.

e) Lesões condrais - Devem ser lembradas quando os atletas apresentam dores crônicas na articulação do ombro e do cotovelo. Em determinadas situações, podem fazer com que o esportista sinta estalidos ou até mesmo crepitações durante o gesto esportivo. Especialmente na população em fase de crescimento, devemos lembrar que as osteocondrites podem ser a causa de dor ao redor da articulação - devemos lembrar deste diagnóstico para pesquisar estas lesões, senão elas poderão passar despercebidas no exame clínico inicial do atleta.

\section{CONCLUSÕES}

Como pode ser observado, as lesões do membro superior no esporte podem fazer parte do dia a dia de qualquer médico, até mesmo daqueles que não cuidam exclusivamente de tenistas. As tendinopatias, comuns destas regiões, devem ser objeto de estudo, para que possamos evitar cada vez mais a sua forma crônica, o que pode fazer com que o paciente necessite de tratamentos mais demorados e custosos para o sistema de saúde.

Esperamos que este artigo auxilie o leitor na condução destas patologias, visando o melhor cuidado ao paciente esportista e não esportista. A literatura revisada foi extensa, e a leitura complementar dos artigos referenciados neste trabalho completará com detalhes as informações aqui apresentadas. 


\section{REFERÊNCIAS}

1. Silva RT. Lesões esportivas no tênis. In: Cohen M, Jorge RA. Lesões nos esportes: diagnóstico, prevenção e tratamento. Rio de Janeiro: Revinter; 2002. p. 709-32.

2. Hill JA. Epidemiologic perspective on shoulder injuries. Clin Sports Med. 1983;2(2):241-6.

3. Schneider S, Seither B, Tönges S, Schmitt H. Sports injuries: population based representative data on incidence, diagnosis, sequelae, and high risk groups. $\mathrm{Br}$ J Sports Med. 2006;40(4):334-9.

4. Bigliani LU, Morrison DS, April EW. The morphology of the acromion and its relation to rotator cuff tears. Orthop Trans. 1986;10:228.

5. Zuckerman JD, Kummer FJ, Cuomo F. The influence of coracoacromial arch anatomy on rotator cuff tears. J Shoulder Elbow Surg. 1992;19(1):4-13.

6. Doneux PS, Miyazaki AN, Pinheiro Jr JA, Funchal LFZ, Checchia SL. Tratamento da síndrome do impacto em tenistas. Rev Bras Ortop. 1998;33(12):939-44.

7. Jobe CM, Pink MM, Jobe FW, Shaffer B. Anterior shoulder instability, impingement, and rotator cuff tear: theories and concepts. In: Jobe FW, editor. Operative techniques in upper extremity sports injuries. St. Louis: Mosby; 1996. p.164-76.

8. Kibler WB. The role of the scapula in athletic shoulder function. Am J Sports Med. 1998;26(2):325-37.

9. Kibler WB, Uhl TL, Maddux JW, Brooks PV, Zeller B, McMullen J. Qualitative clinical evaluation of scapular dysfunction: a reliability study. J Shoulder Elbow Surg. 2002;11(6):550-6.

10. DiGiovine NM, Jobe FW, Pink M, Perry J. An electromyographic analysis of the upper extremity in pitching. J Shoulder Elbow Surg.1992(1):15-22.

11. Kibler WB. Biomechanical analysis of the shoulder during tennis activities. Clin Sports Med. 1995;14(1):79-85.

12. Arroyo JS, Hershon SJ, Bigliani LU. Special considerations in the athletic throwing shoulder. Orthop Clin North Am. 1997;28(1):69-78.

13. Abrams JS. Special shoulder problems in the throwing athlete: pathology, diag nosis, and nonoperative management. Clin Sports Med. 1991;10(4):839-61.

14. Lehman RC. Shoulder pain in the competitive tennis player. Clin Sports Med.1988; 7(2):309-27.

15. Rudzki JR, Shaffer B. New approaches to diagnosis and arthroscopic management of partial-thickness cuff tears. Clin Sports Med. 2008;27(4):691-717.

16. Weber SC. Arthroscopic debridement and acromioplasty versus mini-open repair in the treatment of significant partial-thickness rotator cuff tears. Arthros copy. 1999;15(2):126-31.

17. Ellenbecker TS, Roetert EP, Bailie DS, Davies GJ, Brown SW. Glenohumeral joint total rotation range of motion in elite tennis players and baseball pitchers. Med Sci Sports Exerc. 2002;34(12):2052-6.

18. Crockett HC, Gross LB, Wilk KE, Schwartz ML, Reed J, O'Mara J, et al. Os seous adaptation and range of motion at the glenohumeral joint in professiona baseball pitchers. Am J Sports Med. 2002;30(1):20-6.

19. Lintner D, Mayol M, Uzodinma O, Jones R, Labossiere D. Glenohumeral internal rotation deficits in professional pitchers enrolled in an internal rotation stretching program. Am J Sports Med. 2007;35(4):617-21

20. Snyder SJ, Karzel RP, Del Pizzo W, Ferkel RD, Friedman MJ. SLAP lesions of the shoulder. Arthroscopy. 1990;6(4):274-9.

21. Powell SE, Nord KD, Ryu RK. The diagnosis, classification, and treatment of SLAP lesions. Oper Tech Sports Med. 2004;12(1):99-110.

22. Barber FA, Field LD, Ryu RK. Biceps tendon and superior labrum injuries: decision making. Instr Course Lect. 2008;57:527-38.

23. Paxinos A, Walton J, Rütten S, Müller M, Murrell GA. Arthroscopic stabilization of superior labral (SLAP) tears with biodegradable tack: outcomes to 2 years. Arthroscopy. 2006;22(6):627-34.

24. Rhee YG, Lee DH, Lim CT. Unstable isolated SLAP lesion: clinical presentation and outcome of arthroscopic fixation. Arthroscopy. 2005;21(9):1099.

25. Franceschi F, Longo UG, Ruzzini L, Rizzello G, Maffulli N, Denaro V. No advantages in repairing a type II superior labrum anterior and posterior (SLAP) lesion when associated with rotator cuff repair in patients over age 50: a randomized controlled trial. Am J Sports Med. 2008;36(2):247-53.

26. Voos JE, Pearle AD, Mattern CJ, Cordasco FA, Allen AA, Warren RF. Outcomes of combined arthroscopic rotator cuff and labral repair. Am J Sports Med. 2007;35(7):1174-9

27. Silva RT, Hartmann LG, Laurino CF, Biló JP. Clinical and ultrasonographiccorrelation between scapular dyskinesia and subacromial space measurement amongjunior elite tennis players. Br J Sports Med. 2008. [Epub ahead of print]

28. RT; Santos MB. Tennis Elbow: a survey among 839 tennis players with and without injury. Med Sci Tennis. 2008;13(1):36-41

29. Bisset L, Paungmali A, Vicenzino B, Beller E. A systematic review and metaanalysis of clinical trials on physical interventions for lateral epicondylalgia. $\mathrm{Br}$ J Sports Med. 2005;39(7):411-22.

30. Roetert EP, Brody H, Dillman CJ, Groppel JL, Schultheis JM. The biomechanics of tennis elbow. An integrated approach. Clin Sports Med. 1995;14(1):47-57.

31. Knudson D, Blackwell J. Upper extremity angular kinematics of the one-handed backhand drive in tennis players with and without tennis elbow. Int J Sports Med. 1997;18(2):79-82

32. Nirschl RP, Ashman ES. Elbow tendinopathy: tennis elbow. Clin Sports Med. 2003;22(4):813-36

33. Chumbley EM, O'Connor FG, Nirschl RP. Evaluation of overuse elbow injuries. Am Fam Physician. 2000;61(3):691-700.

34. Tuite MJ, Kijowski R. Sports-related injuries of the elbow: an approach to MRI interpretation. Clin Sports Med. 2006;25(3):387-408.

35. Bencardino JT, Rosenberg ZS. Entrapment neuropathies of the shoulder and elbow in the athlete. Clin Sports Med. 2006;25(3):465-87.

36. Johnson GW, Cadwallader K, Scheffel SB, Epperly TD. Treatment of lateral epicondylitis. Am Fam Physician. 2007;76(6):843-8.

37. Stasinopoulos D, Stasinopoulou K, Johnson MI. An exercise programme for the management of lateral elbow tendinopathy. $\mathrm{Br} \mathrm{J}$ Sports Med. 2005;39(12):944-7.

38. Newcomer KL, Laskowski ER, Idank DM, McLean TJ, Egan KS. Corticosteroid injection in early treatment of lateral epicondylitis. Clin J Sport Med. 2001;11(4):214-22

39. Hay EM, Paterson SM, Lewis M, Hosie G, Croft P. Pragmatic randomized controlled trial of local corticosteroid injection and naproxen for treatment of lateral epicondylitis of elbow in primary care. BMJ. 1999;319(7215):964-8.

40. Radwan YA, EISobhi G, Badawy WS, Reda A, Khalid S. Resistant tennis elbow: shock-wave therapy versus percutaneous tenotomy. Int Orthop. 2008;32(5):671-7.

41. Lebrun CM. Low-dose extracorporeal shock wave therapy for previously untreated lateral epicondylitis. Clin J Sport Med. 2005;15(5):401-2.

42. Chung B, Wiley JP. Effectiveness of extracorporeal shock wave therapy in the treatment of previously untreated lateral epicondylitis: a randomized controlled trial. Am J Sports Med. 2004;32(7):1660-7.

43. Mishra A, Woodall J Jr, Vieira A. Treatment of tendon and muscle using platelet-rich plasma. Clin Sports Med. 2009;28(1):113-25

44. Mishra A, Pavelko T. Treatment of chronic elbow tendinosis with buffered platelet-rich plasma. Am J Sports Med. 2006;34(11):1774-8.

45. Silva RT. Treatment of chronic tennis elbow with platelet-rich plasma. Case Report. Adv Orthop. 2009;1(3):108-11.

46. Connell DA, Ali KE, Ahmad M, Lambert S, Corbett S, Curtis M. Ultrasound-guided autologous blood injection for tennis elbow. Skeletal Radiol. 2006;35(6):371-7.

47. Perkins RH, Davis D. Musculoskeletal injuries in tennis. Phys Med Rehabil Clin N Am. 2006;17(3):609-31.

48. Silva RT, Hartmann LG, Laurino CF. Stress reaction of the humerus in tennis players. Br J Sports Med. 2007;41(11):824-6.

49. Silva RT, Cohen M, Matsumoto MH, Gracitelli GC. Avaliação das lesões ortopédicas em tenistas amadores competitivos. Rev Bras Ortop. 2005;40(5):270-9. 